\title{
Essais
}

ESSAIS

Revue interdisciplinaire d'Humanités

Hors-série 6 | 2021

Agrobiodiversité et territoires

\section{Contribution des ressources génétiques animales aux services écosystémiques liés au développement territorial}

Contribution of animal genetic resources to ecosystem services linked to territorial development

Grégoire Leroy et Étienne Verrier

\section{OpenEdition}

Journals

Édition électronique

URL : https://journals.openedition.org/essais/7438

DOI : 10.4000/essais.7438

ISSN : 2276-0970

Éditeur

École doctorale Montaigne Humanités

Édition imprimée

Date de publication : 1 mars 2021

Pagination : 79-87

ISBN : 978-2-492780-00-4

ISSN : 2417-4211

Référence électronique

Grégoire Leroy et Étienne Verrier, «Contribution des ressources génétiques animales aux services écosystémiques liés au développement territorial », Essais [En ligne], Hors-série 6 | 2021, mis en ligne le 16 mars 2021, consulté le 18 janvier 2023. URL : http://journals.openedition.org/essais/7438; DOI : https://doi.org/10.4000/essais.7438 


\title{
Contribution des ressources génétiques animales aux services écosystémiques liés au développement territorial
}

\author{
Grégoire Leroy, Étienne Verrier
}

L'élevage est au cœur de multiples enjeux de durabilité, que l'on considère la sécurité alimentaire, la lutte contre la pauvreté, ou le changement climatique. L'activité d'élevage est aussi liée à une diversité de services écosystémiques rendus au sein des agroécosystèmes à une très large échelle. À l'heure actuelle, sur les 2,5 milliards d'ha de terres agricoles utilisées par l'élevage dans le monde, 2 milliards correspondent à des espaces pâturés, dont 1,3 ne peuvent pas être valorisés par autre chose que de l'activité pastorale ${ }^{1}$. Le nombre de personnes pratiquant le pastoralisme dans le monde est estimé entre 200 et 500 millions d'individus ${ }^{2}$. En comparaison de systèmes plus intensifs, les agroécosystèmes pastoraux font généralement appel à des espèces et des races autochtones ${ }^{3}$, relativement peu productives, mais disposant de caractères d'adaptation les rendant aptes à tirer parti des contraintes de l'environnement et du système de production local. Au cours des dernières décennies, l'intensification des systèmes d'élevage dans de nombreux pays a eu pour conséquence un remplacement progressif de ces populations par des races internationalement reconnues et a priori plus productives.

Dans le cadre d'une réflexion sur les services écosystémiques fournis par les agroécosystèmes impliquant l'élevage de manière générale, et les systèmes pastoraux en particulier, il apparaît légitime de s'interroger sur le lien entre les ressources génétiques utilisées, et les services fournis. Nous nous proposons tout d'abord de revenir sur la notion de ressource génétique en tant que telle,

1 Anne Mottet, Cees de Haan, Alessandra Falcucci, Giuseppe Tempio, Carolyn Opio, Pierre Gerber, "Livestock: On our plates or eating at our table? A new analysis of the feed/food debate", Global Food Security, 14, 2017, p. 1-8.

2 FAO, Crossing boundaries: Legal and policy arrangements for cross-border pastoralism, FAO/IUCN, Rome, 2018.

3 Gregoire Leroy, Roswitha Baumung, Paul Boettcher, Beate Scherf, Irene Hoffmann, "Sustainability of crossbreeding in developing countries; definitely not like crossing a meadow...", Animal, 10, 2016, p. 262-273. 
avant d'étudier en quoi le choix d'une ressource peut affecter un ou des services écosystémiques. À partir de là nous nous intéresserons à la manière dont ces services sont actuellement perçus, et quels sont les enjeux et contraintes pour leur fourniture.

\section{Que sont les ressources génétiques animales (RGAs)?}

La Convention pour la Diversité Biologique de 1992 définit une ressource génétique comme un "matériel d'origine végétale, animale, microbienne ou autre, contenant des unités fonctionnelles de l'hérédité" ou comme "un matériel génétique ayant une valeur effective ou potentielle ", les RGAs se rapportant plus spécifiquement aux espèces animales domestiquées par l'homme.

En termes pratiques, cette définition implique que les ressources génétiques doivent être considérées à différentes échelles, du gène à l'espèce. En ce qui concerne les animaux domestiques, il conviendra plus particulièrement de considérer l'échelle populationnelle ou raciale. Le concept de race animale intègre des dimensions tant socio-culturelles que génétiques, et il s'agit de l'unité organisationnelle la plus largement utilisée dans la gestion des ressources génétiques animales. On répertorie à l'heure actuelle près de 8200 races appartenant à 38 espèces dans le monde ${ }^{4}$. Parmi celles-ci, 7127 sont considérées comme locales au sens de la FAO, c'est-à-dire ne sont répertoriées que dans un seul pays, tandis que 1048 sont dites transfrontalières. Ainsi, la race bovine Holstein est présente dans au moins 163 pays du monde, en général au sein de systèmes mixtes ou intensifs. Une autre manière de classer les races consiste à différencier à l'échelle des pays, les populations importées, dont la Holstein fait souvent partie, des races localement adaptées, c'est-à-dire des populations autochtones ou implantées depuis suffisamment longtemps pour être adaptées à l'environnement et au système de production local. Les races localement adaptées peuvent être par ailleurs transfrontalières, notamment certaines races de ruminants utilisées dans les systèmes transhumants, qui sont particulièrement importantes au regard des services écosystémiques fournis par le pastoralisme.

\section{Quels liens entre les RGAs et les services écosystémiques territoriaux?}

La place des ressources génétiques dans le cadre conceptuel des services écosystémiques n'est pas forcement bien définie au sein de la littérature. Si pour Zhang et al. ${ }^{5}$, la diversité génétique des plantes cultivées et des animaux d'élevage constitue un service écosystémique en tant que tel, il a été souligné

4 DAD-IS, Domestic Animal Diversity Information System, FAO, Rome, http://www.fao.org/ dad-is/en/ (consulté le 20/02/2020).

5 W. Zhang, T. H. Ricketts, C. Kremen, K. Carney, S. M. Swinton, Ecosystem services and dis-services to agriculture, Écol. Écon., 64, 2007, p. 253-60. 
que la biodiversité (dans laquelle s'intègre les RGAs) pouvait aussi être vue comme un régulateur des processus écosystémiques ${ }^{6}$. Enfin, pour Ovaska et Soini ${ }^{7}$, les races domestiques seraient des fournisseurs de services écosystémiques, tandis que Marsoner et al. ${ }^{8}$ (2018) ont suggéré de quantifier le nombre de races autochtones comme indicateurs des services culturels.

En pratique, il peut être considéré que la diversité des RGAs va influencer les services écosystémiques fournis par les agroécosystèmes au travers des systèmes de production et des choix zootechniques, par exemple le fait d'utiliser un ou plusieurs génotypes, races, espèces. Les individus ainsi utilisés exprimeront, pour des caractères donnés, des phénotypes spécifiques, susceptibles d'influencer, voire de générer, les services écosystémiques de l'agroécosystème. L'expression des phénotypes en question peut être liée à un gène unique (déterminisme monogénique) ou au contraire une combinaison de gènes (déterminisme multifactoriel). Comme l'illustre la Figure 1, la diversité des RGAs est en mesure d'impacter de nombreux services et disservices (fonctions de l'écosystème perçues comme négatives) écosystémiques, que l'on considère les services culturels, d'approvisionnement, ou de régulation. Ces impacts peuvent exister tant à l'échelle du gène, que de la race ou de l'espèce sélectionnée par l'éleveur. Par exemple, le gène Booroola chez le mouton est connu pour agir fortement sur la prolificité des brebis, impactant ainsi les services d'approvisionnement correspondants.

Tableau 1 : Exemples de phénotypes impactant les services écosystémiques liés au territoire

\begin{tabular}{|c|c|c|c|}
\hline $\begin{array}{c}\text { Services } \\
\text { écosystémiques }\end{array}$ & Phénotypes & Modes d'expression & Échelles considérées \\
\hline Multiples & Adaptation à l'altitude & $\begin{array}{c}\text { Complexe } \\
\text { (Divers QTLs } \\
\text { et gènes identifiés) }\end{array}$ & $\begin{array}{l}\text { Espèces (Yak), races } \\
\text { (Chèvre tibetaine...) }\end{array}$ \\
\hline Multiples & $\begin{array}{c}\text { Pelage lisse et court } \\
\text { favorisant l'adaptation } \\
\text { à la chaleur }\end{array}$ & $\begin{array}{l}\text { Monogenique } \\
\text { (gène slick) }\end{array}$ & $\begin{array}{c}\text { Races (races bovines } \\
\text { Senepol et créoles) et sous- } \\
\text { populations (bovin Holstein) }\end{array}$ \\
\hline Entretien de l'habitat & Aptitude à la marche & $\begin{array}{c}\text { Complexe } \\
\text { (QTLs identifiés) }\end{array}$ & $\begin{array}{c}\text { Espèces, races, individus } \\
\text { (race ovine Border Leicester, } \\
\text { chèvres marocaines) }\end{array}$ \\
\hline $\begin{array}{c}\text { Gestion de } \\
\text { l'embroussaillement }\end{array}$ & $\begin{array}{c}\text { Préférence alimentaire pour } \\
\text { l'alimentation fibreuse }\end{array}$ & Complexe & Race (Chèvre Damascus) \\
\hline $\begin{array}{l}\text { Érosion et maintien } \\
\text { de la biodiversité }\end{array}$ & $\begin{array}{l}\text { Préférences alimentaires } \\
\text { et comportement } \\
\text { de piétinement }\end{array}$ & Complexe & Espèces \\
\hline
\end{tabular}

6 Georgina M. Mace, Ken Norris, Alastair H. Fitter, "Biodiversity and ecosystem services: a multilayered relationship", Trends in Ecology \& Evolution, 27, 2012, p. 19-26.

7 Thomas Marsoner, Lukas Egarter Vigl, Frederick Manck, Gunter Jaritz, Ulrike Tappeiner, Erich Tasser, "Indigenous livestock breeds as indicators for cultural ecosystem services: A spatial analysis within the Alpine Space", Ecological indicators, 94, 2018, p. 55-63.

8 Ulla Ovaska, Katriina Soini, "Native breeds as providers of ecosystem services: the stakeholders' perspective", Trace: Finnish Journal of Human-Animal Studies, 2, 2016. 


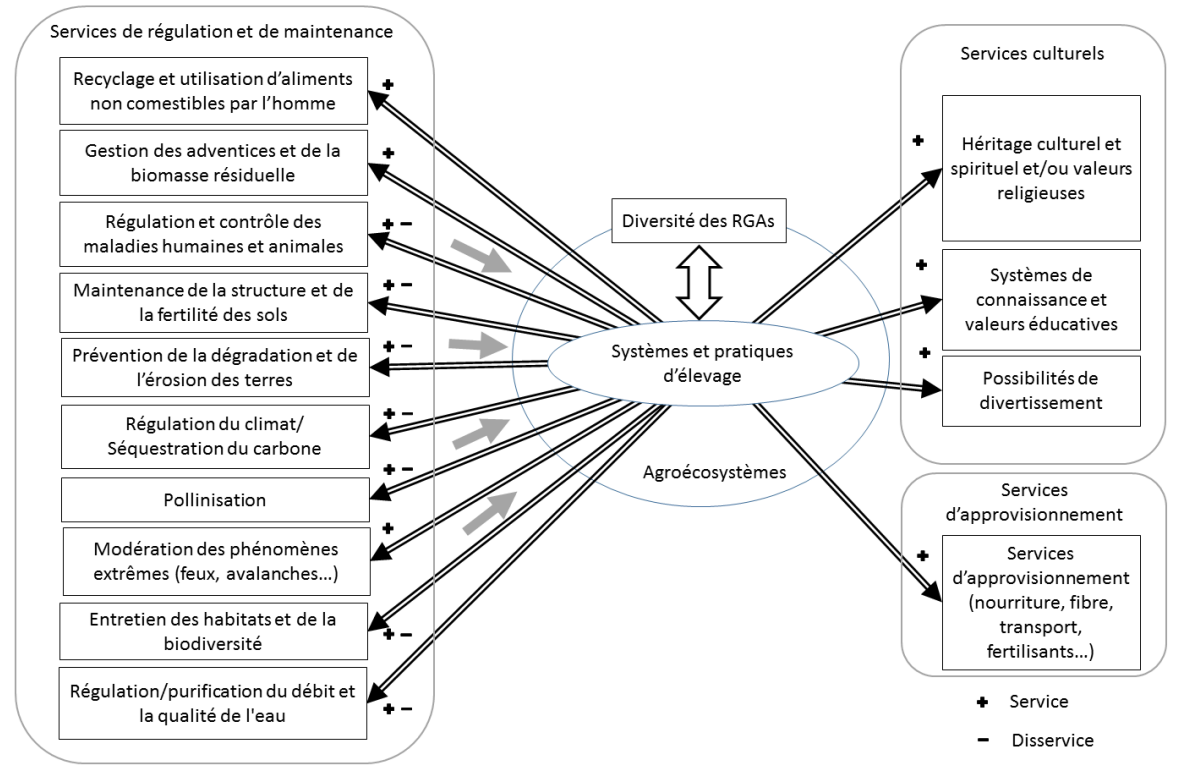

Figure 1 : Impact de la diversité des RGA sur les services écosystémiques aux travers des systèmes et pratiques d'élevage (adapté de Leroy et al. ${ }^{9}$ )

En ce qui concerne les services de régulation en lien avec l'entretien des territoires, les caractères d'adaptation tiennent une importance particulière, et permettent aux animaux de faire usage d'un territoire particulier, qui ne pourraient être valorisé par d'autres individus non adaptés à ceux-ci (Tableau 1). Ainsi certaines espèces (Yak, Chameau ou Dromadaire) ou races (la race bovine Yakut en Sibérie, par exemple) se révèlent particulièrement adaptées à des environnements extrêmes. Certaines races bovines telles que la Senepol possèdent une pilosité qui favorise la sudation et la résistance à la chaleur, en lien avec une mutation au gène Slick, mutation qui a par ailleurs été introgressée au sein de certaines sous-populations de la race Holstein ${ }^{10}$. Les aptitudes spécifiques de certaines espèces ou races pour parcourir de longues distances permettent d'élargir le territoire entretenu par l'activité pastorale. On notera par ailleurs que les éleveurs de bovins laitiers ont la possibilité de sélectionner leurs animaux sur cette dernière aptitude, au travers de certains caractères morphologiques inclus dans les objectifs de sélection comme les aplombs. L'importance de l'adaptation est largement mise en évidence par le nombre d'échecs dans les projets d'introgression et de croisement dans les

9 Gregoire Leroy, Roswitha Baumung, Paul Boettcher, Badi Besbes, Tatiana From, Irene Hoffmann, "Animal genetic resources diversity and ecosystem services", Global food security, 17, 2018, p. 84-91.

10 S. Dikmen, F. A. Khan, H. J. Huson, T. S. Sonstegard, J. I. Moss, G. E. Dahl, P. J. Hansen, "The SLICK hair locus derived from Senepol cattle confers thermotolerance to intensively managed lactating Holstein cows", Journal of dairy science, 97, 2014, p. 5508-5520. 
pays en développement, du fait d'un manque d'adaptation des génotypes ainsi importés aux environnements locaux ${ }^{11}$. À ce titre, les approches de type "landscape genomics" ont permis de mettre en évidence un nombre croissant de variants génétiques impliqués dans l'adaptation des races à leurs milieux ${ }^{12}$.

Pour d'autres races comme la chèvre Damascus, des comportements alimentaires spécifiques, en l'occurrence une préférence pour les fibres riches en tannin, peut se révéler un atout pour le contrôle de l'embroussaillement. En ce qui concerne certains services comme le maintien de la biodiversité herbagère, s'il a pu être démontré que celle-ci était largement impactée par l'espèce ou la combinaison d'espèces utilisées pour pâturer, les études ne semblent pas avoir mis en évidence de différences selon le choix de la race ${ }^{13}$ (Rook et al., 2004), le chargement restant généralement le principal facteur d'impact sur cette biodiversité.

Les antagonismes souvent observés entres services d'approvisionnement et services de régulation et de maintenance se retrouvent quand on regarde les caractères d'intérêt pour la selection avec des corrélations génétiques généralement négatives entre caractères de productivité (production laitière, croissance...) et caractères fonctionnels (aptitude à la reproduction, résistance au stress thermiques ou aux maladies). Cependant, la combinaison de différentes races peut se révéler intéressante pour la fourniture de services écosystémiques de types différents. À titre d'exemple, le croisement de femelles Aubrac élevées en système herbagers du sud du Massif central avec des taureaux de races bouchères, telles que la Charolaise, permet de prendre avantage de la rusticité de la première race pour exploiter un environnement difficile (services de régulation et de maintenance), tout en produisant des veaux de croissance améliorée recherchés sur le marché (services d'approvisionnement). Ainsi si l'on considère que les ressources génétiques employées relèvent du choix zootechnique, au même titre que le chargement pastoral, celles-ci constituent à ce titre un facteur de régulation des services fournis par l'agroécosystème.

11 Gregoire Leroy, Irene Hoffmann, Tatiana From, Sipke Joost Hiemstra, Gustavo Gandini, "Perception of livestock ecosystem services in grazing areas inside and outside Europe", Animal, 12, 2018, p. 2627-2638.

12 Badr Benjelloun, Diversité des génomes et adaptation locale des petits ruminants d'un pays méditerranéen: le Maroc, thèse de doctorat, 2015.

13 AJ Rook, Bertrand Dumont, Johaness Isselstein, Koldo Osoro, Michiel WalliesDeVries, Giuseppe Parente, Jane Mills, "Matching type of livestock to desired biodiversity outcomes in pastures -a review", Biological Conservation, 119, 2004, p. 137-150. 


\section{Perceptions, enjeux et contraintes autour des services écosysté- miques territoriaux liés à l'élevage et aux RGAs}

L'un des principaux enjeux autour des services écosystémiques non marchands, tels que ceux liés à l'entretien du territoire, se situe autour de la valeur et de la perception qu'ont les différents acteurs de ces services. Dans l'objectif de mieux comprendre les facteurs impactant la perception des services liés à l'activité de pâturage (approvisionnement non compris), 82 cas d'étude issus de pays européens et non-européens, fournis par différentes catégories d'experts (chercheurs, ONG, associations d'éleveurs...) ont été collectés en 2013 et analysés ${ }^{14}$. De ces différents cas (Figure 2 [I]), il est apparu que la perception était apparue particulièrement positive pour les services liant la culture et les valeurs de l'environnement naturel et des paysages, d'autres services, tels que l'entretien de l'habitat, le contrôle des résidus, adventices ou de l'embroussaillement étant aussi perçus tout à fait positivement.

L'utilisation d'analyses multivariées de type ACP (analyse en composantes principales), pour identifier les facteurs impactant la perception des services, a notamment permis de mettre en évidence une corrélation généralement positive de la perception des différents types de service (les services d'approvisionnement n'étant pas considérés) (Figure 2 [IIa]). Par ailleurs, la perception est apparue plus positive au sein des pays européens, dans les cas d'étude utilisant une combinaison d'espèces, ainsi que dans les espaces protégés (Figure $\mathbf{2}$ [II b et c]), soulignant l'intérêt potentiel du pâturage mixte.

Parmi les cas non-européens, les principales contraintes limitant la fourniture des services écosystémiques fournis par l'élevage sont apparues liées à un manque de reconnaissance de ces services ainsi qu'à l'absence de politiques et de cadre réglementaire supportant ceux-ci. Par contraste, l'existence d'un cadre dédié à l'échelle européenne (règlement européen " agroenvironnemental »du 30 juin 1992) explicite la reconnaissance juridique de ces services pour les cas européens. La principale contrainte identifiée pour ceux-ci correspond au manque de revenu lié à l'activité d'élevage, illustrant notamment les enjeux autour de la quantification, notamment financière, des services écosystémiques.

14 Gregoire Leroy, Irene Hoffmann, Tatiana From, Sipke Joost Hiemstra, Gustavo Gandini, "Perception of livestock ecosystem services in grazing areas inside and outside Europe", Animal, 12, 2018, p. 2627-2638. 
(I)

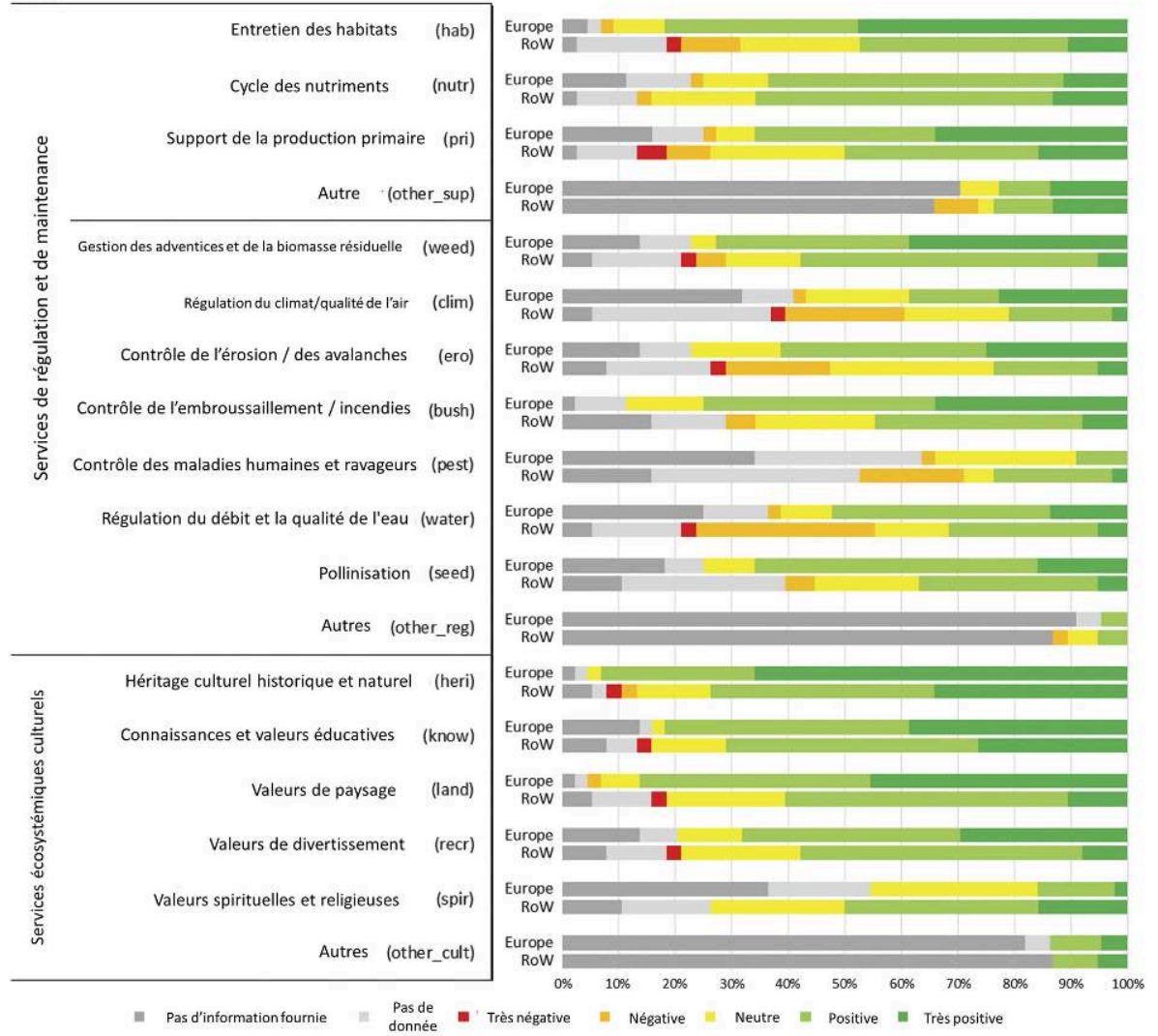

(II)

(a)

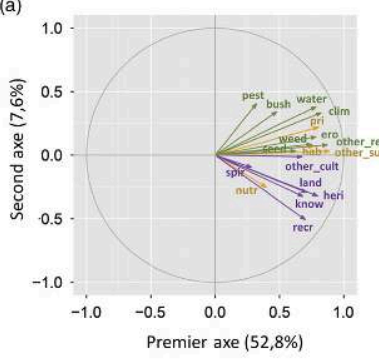

Régulation Support Culturel (b)

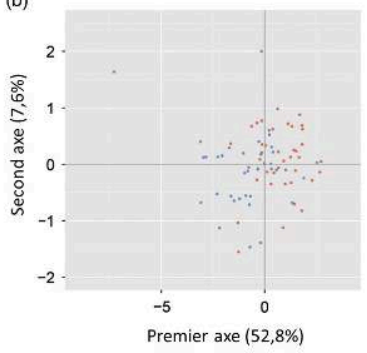

Europe Autres pays (RoW)

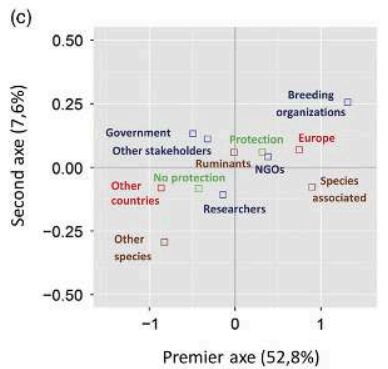

Aires protégées Espèces Localisation Acte

Figure 2 : Perception des services selon les cas européens et non-européens (I), et projections de variables, études de cas et facteurs explicatifs sur les deux premiers axes de l'analyse factorielle multiples (II) avec (a) Cercle de corrélation des services écosystémiques en tant que variables quantitatives ; (b) projection des 82 études de cas analysées ; (c) projection des facteurs explicatifs. 
Les choix qui peuvent être faits par les éleveurs en termes d'espèces, de races et d'animaux sont en mesure d'impacter largement les différents services écosystémiques fournis par les agroécosystèmes, y compris ceux liés à l'entretien des paysages, tout particulièrement dans leur dimension culturelle, l'animal faisant partie intégrante de ces paysages. Un enjeu majeur autour $\mathrm{du}$ lien entre les ressources utilisées et les services rendus reste de manière générale, et particulièrement pour les pays non-européens, le manque de reconnaissance de ces services. Une autre difficulté porte sur la quantification (notamment financière) de ce lien, soulignant la nécessité d'études multidisciplinaires qui faciliteront la prise en compte de ces services dans les choix zootechniques auxquels sont confrontés les éleveurs.

\section{Grégoire Leroy}

Animal Genetic Resources Branch, Animal Production and Health Division, Food and Agriculture Organization of the United Nations (FAO) 00153, Rome, Italy Université Paris-Saclay, Inrae, AgroParisTech, UMR GABI, 78350 Jouy-en-Josas gregoire.leroy@fao.org

Étienne Verrier Université Paris-Saclay, Inrae, AgroParisTech, UMR GABI, 78350 Jouy-en-Josas etienne.verrier@agroparistech.fr

Grégoire Leroy est titulaire d'une thèse en génétique animale obtenue en 2008. Maître de conférences au sein de l'UMR INRAE/AgroParisTech Génétique Animale et Biologie Intégrative, il est actuellement mis à disposition depuis 2015 auprès de l'Organisation des Nations Unies pour l'Alimentation et l'Agriculture. Ses thématiques de recherches portent sur la caractérisation, la conservation et la gestion durable des ressources génétiques animales.

Étienne Verrier est professeur de génétique animale à AgroParisTech et directeur-adjoint de l'UMR GABI (Inrae/AgroParisTech). Ses travaux portent sur la définition d'indicateurs pour la gestion génétique des populations animales et la valorisation et l'inscription territoriale des races locales. 


\section{Résumé}

Au travers des systèmes de productions au sein desquels celles-ci s'intègrent, les races et espèces d'élevage contribuent à une diversité de services écosystémiques, dont plusieurs impactent le développement des territoires, notamment dans leur dimension culturelle. Ces services sont perçus de manière différente en fonction de la région, du répondant, du statut de protection du territoire ou l'espèce considérée. Le manque de reconnaissance de ces services, en particulier au sein des pays non-européens, constitue un enjeu majeur pour leur pérennité et celle des agroécosystèmes correspondants.

\section{Mots-clés}

Ressources génétiques animales, services écosystémiques, race, territoire, pâturage.

\section{Abstract}

Through the production systems where they are raised, the breeds and livestock species contribute a diversity of ecosystem services, many of which impact the development of territories, particularly in their cultural dimension. These services are perceived differently depending on the region, the respondent, the protection status of the territory or the species considered. The lack of recognition of these services, particularly in non-European countries, constitutes a major challenge for the sustainability of the corresponding agro-ecosystems.

\section{Keywords}

Animal genetic resources, ecosystem service, animal breed, territory, pasture. 